\title{
Recruitment of an Area Involved in Eye Movements During Mental Arithmetic
}

André Knops, ${ }^{1,2,3}$ * Bertrand Thirion,, ${ }^{2,4}$ Edward M. Hubbard, ${ }^{1,2,3}$ Vincent Michel, ${ }^{2,3,4}$ Stanislas Dehaene ${ }^{1,2,3,5}$

${ }^{1}$ INSERM, Cognitive Neuroimaging Unit, F-91191 Gif-sur-Yvette, France. ${ }^{2}$ CEA, I2BM, NeuroSpin, F-91191 Gif-sur-Yvette, France. ${ }^{3}$ Université Paris-Sud, F-91405 Orsay, France. ${ }^{4}$ INRIA Saclay - Île de France, Orsay, France. ${ }^{5}$ Collège de France, Paris, France.

*To whom correspondence should be addressed. E-mail: knops.andre@gmail.com

Throughout the history of mathematics, concepts of number and space have been tightly intertwined. We tested the hypothesis that cortical circuits for spatial attention contribute to mental arithmetic. We trained a multivariate classifier to infer the direction of an eye movement, left or right, from the brain activation measured in posterior parietal cortex. Without further training, the classifier then generalized to an arithmetic task. Its left versus right classification could be used to sort out subtraction versus addition trials, whether performed with symbols or with sets of dots. These findings are consistent with the suggestion that mental arithmetic co-opts parietal circuitry associated with spatial coding.

The human species is unique in its capacity to create revolutionary cultural inventions such as writing and mathematics, which dramatically enhance its native competence. From a neurobiological standpoint, such inventions are too recent for natural selection to have dedicated them specific brain mechanisms. It has therefore been suggested that they co-opt or "recycle" evolutionarily older circuits with a related function (1), thus enriching (without necessarily replacing) their domain of use. For instance, learning to read recruits a left infero-temporal area originally engaged in object recognition, and even the seemingly arbitrary shapes of our letters may originate in a neural repertoire of junctions detectors useful for scene recognition and available to all primates (2). In the case of mathematics, although foundational intuitions such as number sense (3) and spatial maps (4) are present in many animal species and in humans prior to education, mathematical constructions vastly exceed these initial domains of inherited competence. It has been argued that analogies between number and space play a crucial role in the expansion of mathematical concepts (5). Here, we investigate the role of brain areas for spatial coding in mental arithmetic.

Many behavioral experiments have demonstrated automatic links between number and space. Even young children and uneducated adults readily conceive of numbers as forming an internal spatial continuum or "mental number line” (6). Merely perceiving an Arabic digit suffices to elicit a spatial bias in both attentional orienting (7) and manual responses (8), with small numbers inducing a left-sided and large numbers a right-sided advantage in left-to-right readers. When adults perform approximate additions and subtractions, they overshoot towards larger numbers for addition and towards smaller numbers for subtraction, as if carried along by spatial momentum (9). Perhaps the most conclusive evidence for numerical-spatial links comes from the syndrome of spatial hemineglect, in which brain-lesioned patients fail to attend to one side of space, usually the left side. When such patients attempt to bisect a numerical interval, their responses are shifted towards larger numbers, as if neglecting the left half of the numerical segment where small numbers are represented (10).

The brain mechanisms of these numerical-spatial interactions, however, remain largely unknown. In both monkeys and humans, number processing recruits a brain area deep within the intraparietal sulcus (hIPS) $(11,12)$. This site partially overlaps with area ventral intraparietal cortex (VIP), an area coding for multimodal spatial movement and tightly interconnected with nearby area lateral intraparietal cortex (LIP) involved in saccadic and attention control (1315). A model of the VIP-LIP circuitry proposes that it implements a form of vector addition of eye and retinal position information (16). We therefore reasoned that this circuit might be co-opted for a similar function in the arithmetic domain. Given the cultural link between small numbers and the left side of space, and right numbers and the right side of space in left-to-right readers, we predicted that mental addition, which increases number size, would be associated with a rightward shift of attention, and subtraction with a leftward shift. Hence, the activation pattern in parietal cortex during addition would resemble the activation pattern associated with a rightward eye movement, while subtraction would resemble a leftward eye movement.

In a 3 Tesla fMRI scanner, participants first performed a localizer task for eye movements. By contrasting eye 
movements against fixation, we isolated a set of six cortical regions classically associated with saccades and used in all subsequent classifier-based analyses (17): bilateral posterior superior parietal lobule (PSPL), at a site overlapping with the proposed human homolog of monkey area LIP (18); bilateral frontal eye fields proper (FEF); and two clusters of activation lateral to FEF (IFEF; Fig. 1B).

Participants performed a second set of fMRI runs during which they either moved their eyes rightward or leftward on randomly intermixed trials. We adopted a machine learning approach to search for a linear combination of these voxelbased activation signals that reliably separated leftward and rightward saccades (19). We trained a linear support vector machine (SVM) using a ten-fold cross-trial validation approach in which the classifier is first trained on a random subset of $90 \%$ of activation images (one image per trial), and then performance is evaluated on the remaining $10 \%$ of trials. The process was repeated one hundred times, each time with a new random assignment of trials. Using only voxels from the bilateral PSPL region, we obtained a mean accuracy across all participants of $70.3 \% \pm 2.4 \%$ ( 1 standard error), which is significantly above the chance level of $50 \%(t(14)=$ 8.39, $P<.001)$. Analysis by signal detection theory gave similar results (average d' across subjects $=1.1 \pm 0.15, t(14)$ $=7.58, P<.001)$. Thus, saccade direction, which is known to be coded by neurons in monkey area LIP, could be inferred from fMRI of human posterior parietal cortex.

Crucially, we then examined whether the same classifier, without further training, would generalize to approximate arithmetic. In new fMRI runs, participants saw two successive numbers (presented as Arabic numerals or as sets of dots), mentally calculated their approximate sum or difference, and subsequently chose the closest number among 7 possible outcomes. We concentrated on brain activation just after the presentation of the second operand, at which time the participants performed the calculation (Fig. 1A). Calculation activated a network of brain areas comprising bilateral hIPS, prefrontal and premotor areas, with considerable overlap between both notations (Fig. 1B). Calculation overlapped only partially with saccades in bilateral PSPL, but, as predicted, the classifier trained with bilateral PSPL activations during saccades generalized to calculation images. Equating addition with rightward saccades and subtraction with leftward saccades, the mean accuracy for inferring whether an addition or subtraction was performed, averaged over all participants, was $55.0 \% \pm 1.8 \%$, which is significantly greater than chance $(t(14)=2.78, P=$ $\left..015 ; \mathrm{d}^{\prime}=0.31 \pm 0.10, t(14)=2.85, P=.013\right)$.

Further analyses showed that, when the saccades classifier was tested with addition images, it classified them as rightward saccades $61 \%$ of the time (Fig. 2D), which is above chance level $(t(14)=2.35, P=.03)$. For subtraction, however, only $49.1 \%$ of images were classified as leftward saccades $(t(14)=-0.16$, n.s.). This asymmetry, although unexpected, is congruent with earlier reports of larger rightward saccades in response to large numbers, relative to leftward saccades with small numbers (20) and might reflect reading habits in Western cultures.

A key aspect of the cortical recycling view is that saccadic areas of the posterior parietal lobule should contribute to calculation, not only when performed with concrete sets of objects, but even with Arabic numerals, which are a recent product of human culture. We therefore tested the generalization from saccades to calculation in each notation separately. The saccade-trained classifier could distinguish addition from subtraction with an average accuracy of $54.3 \%$ $\pm 2 \%$ for Arabic numerals $(t(14)=2.26, P=.02$; d' $=0.38 \pm$ $0.11, t(14)=2.1, P=.054)$ and with an average accuracy of $55.8 \% \pm 2 \%$ for non-symbolic notation $(t(14)=2.93, P=$ $\left..005 ; \mathrm{d}^{\prime}=0.38 \pm 0.14, t(14)=2.74, P=.016\right)$. Thus, both symbolic and non-symbolic calculations rely in part on brain circuits for saccadic eye movements.

As a further test of this sharing of resources for nonsymbolic and symbolic arithmetic, we also examined the ability to predict which operation was being performed in one notation, on the basis of a classifier trained to sort additions versus subtractions in the other notation. This cross-notation generalization yielded good results, both for the prediction of non-symbolic calculation from the symbolic notation (mean accuracy: $60.7 \% \pm 2.5 \%, t(14)=4.37, P<.001 ; \mathrm{d}^{\prime}=0.53 \pm$ $0.16, t(14)=3.32, P=.005$ ) and vice-versa (mean accuracy: $62.2 \% \pm 2.1 \%, t(14)=5.71, P<.001 ; d^{\prime}=0.75 \pm 0.14, t(14)$ $=5.39, P<.001)$. This finding indicates that the PSPL region is comparably involved in solving mental arithmetic problems in both notations. Approximate arithmetic with sets of dots is part of an inherited 'number sense' available to infants (21) and non-human primates (22), but the cross-notation generalization proves that the corresponding brain circuitry is also used by arithmetic with culturally specific Arabic numerals.

Given the observed parietal cross-talk, one may wonder whether the arithmetic task, although involving only central visual presentations and a constantly present fixation point, led to overt eye movements. Eye position was continuously monitored throughout fMRI, and we found no detectable change in horizontal fixation at or around the time of the arithmetic calculation (17). Furthermore, the observed crosstalk was specific to posterior parietal cortex. Activation patterns in FEF and IFEF could be reliably used to classify left versus right saccades (respectively $56.9 \% \pm 2.4 \%, t(14)=$ $2.87, P=.012$, and $57.8 \% \pm 2.4 \%, t(14)=3.3, P=.005)$, but this classification did not generalize to addition versus subtraction (respectively $49 \% \pm 5.3 \%(t(14)=-0.18, P=.86$ ) and $49.2 \% \pm 6.3 \%(t(14)=-0.12, P=.9))$. The absence of 
decodable FEF activation during arithmetic confirms that calculation specifically engages parietal rather than frontal spatial mechanisms and involves covert visuo-spatial mechanisms, not overt eye movements. As a final test of the specificity of our results to area PSPL, we repeated the major analyses with two control regions (hand motor area M1 and hIPS). None of these regions yielded better-than chance generalization from saccades to calculation (17).

In summary, we demonstrated that a multivariate classifier can distinguish between brain activations during mental addition and subtraction, after having been trained on images from a separate experiment requiring saccades to the right or left. This generalization was observed with numbers presented either as Arabic symbols or as non-symbolic sets of dots, which implies shared cognitive processes between both notations. The observed generalization goes beyond previous demonstrations of classifier-based decoding of line orientation and other pictorial contents from early visual areas (23-26), object identity and category from ventral visual cortex (27), noun identity from distributed cortical regions (28), or intentions from premotor, prefrontal and striatal sites (29). Although generalization was found across different image sizes (27), from real to imagined images (26) or from trained nouns to novel nouns (28), inference remained confined to the trained domain. By contrast, the present research demonstrates generalization from a low-level sensori-motor task to a high-level cognitive task involving learned cultural symbols.

Our results confirm a prediction first made by Hubbard et al. (13) that mental calculation can be likened to a spatial shift along a mental "number line”. In a certain sense, when a Western participant calculates $18+5$, the activation moves "rightward" from 18 to 23. This spatial shift relies on neural circuitry in PSPL shared with those involved in updating spatial information during saccadic eye movements. The findings are reminiscent of the 'embodied cognition' perspective which stipulates that perceptual and action mechanisms lie at the core of human abstract thinking (30). However, the 'recycling' view that we propose does not imply that abstract concepts originate in sensori-motor learning. Indeed, there is ample evidence that abstract numerical concepts have a long evolutionary history and a dedicated neuronal circuitry in intraparietal cortex, partially distinct from neighboring visuo-spatial circuits (31). Our proposal is that human mathematics builds from foundational concepts (space, time, and number) by progressively coopting cortical areas whose prior organization fits with the cultural need. The PSPL area, perhaps because of its capacity for vector addition during eye movement computation (16), appears to have a connectivity or internal structure relevant to arithmetic.
The contribution of PSPL appears to be fundamentally different from the function of other regions such as FEF or hIPS, where no generalization from saccades to calculation was found. The PSPL is active, not only during saccades, but during a broad variety of tasks involving as a common denominator the representation, updating, or attention to spatial locations. This makes it an ideal site for explaining the broad variety of numerical-spatial interactions that have been observed behaviorally with eye, hand, or attention movements (13).

Like any fMRI study, the present work is correlative and cannot establish whether the observed PSPL activation plays a causal role in calculation. One interpretation is that the PSPL is causally recruited during the actual computation of the result of arithmetic operations. Another is that calculation is effected by other means and that the PSPL activation merely reflects a subsequent spread of activation to visuospatial areas, perhaps because the final numerical result attracts attention on the mental number line. To separate those alternatives, future work should evaluate the impact of temporary or permanent lesions, for instance using transcranial magnetic stimulation of dorsal parietal areas, which has already been show to causes joint impairments in attentive visual search and arithmetic (32).

\section{References and Notes}

1. S. Dehaene, L. Cohen, Neuron 56, 384 (2007).

2. M. A. Changizi, Q. Zhang, H. Ye, S. Shimojo, Am. Nat. 167, E117 (2006).

3. S. Dehaene, The Number Sense: How the Mind Creates Mathematics (Oxford Univ.

Press, New York, 1997).

4. C. R. Gallistel, The Organization of Learning (MIT Press, Cambridge, 1990).

5. G. Lakoff, R. E. Nuñez, Where Mathematics Comes From (Basic Books, New York, 2000).

6. S. Dehaene, V. Izard, E. Spelke, P. Pica, Science 320, 1217 (2008).

7. M. H. Fischer, A. D. Castel, M. D. Dodd, J. Pratt, Nature Neurosci. 6, 555 (2003).

8. S. Dehaene, S. Bossini, P. Giraux, J. Exp. Psychol. Gen. 122, 371 (1993).

9. K. McCrink, S. Dehaene, G. Dehaene-Lambertz, Percept. Psychophys. 69, 1324 (2007).

10. M. Zorzi, K. Priftis, C. Umilta, Nature 417, 138 (2002).

11. S. Dehaene, E. Spelke, P. Pinel, R. Stanescu, S. Tsivkin, Science 284, 970 (1999).

12. S. Dehaene, M. Piazza, P. Pinel, L. Cohen, Cogn. Neuropsychol. 20, 487 (2003).

13. E. M. Hubbard, M. Piazza, P. Pinel, S. Dehaene, Nature Rev. Neurosci. 6, 435 (2005).

14. J. R. Duhamel, C. L. Colby, M. E. Goldberg, Science 255, 90 (1992). 
15. C. L. Colby, M. E. Goldberg, Ann. Rev. Neurosci. 22, 319 (1999).

16. A. Pouget, S. Deneve, J. R. Duhamel, Nature Rev. Neurosci. 3, 741 (2002).

17. Materials and methods are available as supporting material on Science Online.

18. M. I. Sereno, S. Pitzalis, A. Martinez, Science 294, 1350 (2001).

19. K. A. Norman, S. M. Polyn, G. J. Detre, J. V. Haxby, Trends Cogn. Sci. 10, 424 (2006).

20. M. H. Fischer, N. Warlop, R. L. Hill, W. Fias, Exp. Psychol. 51, 91 (2004).

21. K. McCrink, K. Wynn, Psychol. Sci. 15, 776 (2004).

22. J. F. Cantlon, E. M. Brannon, PLoS Biol. 5, e328 (2007).

23. J. D. Haynes, G. Rees, Nature Neurosci. 8, 686 (2005).

24. Y. Kamitani, F. Tong, Nature Neurosci. 8, 679 (2005).

25. K. N. Kay, T. Naselaris, R. J. Prenger, J. L. Gallant, Nature 452, 352 (2008).

26. B. Thirion et al., Neuroimage 33, 1104 (2006).

27. E. Eger, J. Ashburner, J. D. Haynes, R. J. Dolan, G. Rees, J. Cogn. Neurosci. 20, 356

(2008).

28. T. M. Mitchell et al., Science 320, 1191 (2008).

29. J. D. Haynes et al., Curr. Biol. 17, 323 (2007).

30. R. L. Goldstone, L. W. Barsalou, Cognition 65, 231

(1998).

31. O. Tudusciuc, A. Nieder, Proc. Natl. Acad. Sci. U. S. A.

104, 14513 (2007).

32. S. M. Göbel, M. F. Rushworth, V. Walsh, Cortex 42, 774 (2006).

33. We thank D. Le Bihan, L. Hertz-Pannier, A. Jobert, and the Neurospin staff for their help. Supported by INSERM, CEA, a McDonnell Foundation centennial fellowship (S.D.), and a Volkswagen Foundation grant (S.D., A.K.).

\section{Supporting Online Material}

www.sciencemag.org/cgi/content/full/1171599/DC1

Materials and Methods

Figs. S1 to S3

References

29 January 2009; accepted 27 April 2009

Published online 7 May 2009; 10.1126/science.1171599

Include this information when citing this paper.

Fig. 1. (A) Schematic depiction of a calculation trial. After the initial presentation of an instructional cue (letters A, S or $\mathrm{C}$ for addition, subtraction or color task, respectively) two quantities were presented successively, either as dot patterns or Arabic digits. After a variable delay period, seven responses alternatives appeared on screen and participants had to choose the alternative closest to the actual outcome. (B) Brain activation in the calculation task and the saccades localizer task projected on lateral and top views of the brain. The images shown result from contrasting symbolic (red) or non-symbolic (green) calculation to the color task, and from contrasting saccades to rest (blue) ( $P=.005$, uncorrected).

Fig. 2. (A) Classification performance (d-prime) for each participant in the saccades task (participants sorted according to d-prime). (B) Classification performance (d-prime) per participant for generalization of the classifier trained on left/right saccades to subtraction/addition trials. (C) Voxel clusters in left and right PSPL region that resulted from the saccade localizer task and served as ROI for the classifier, rendered on white matter/grey matter boundary. (D) Percentages of trials classified as right saccades for subtraction (orange), addition (light blue) and left and right saccades (red and blue, respectively). 
A $\quad$ Classification of left-right saccades in region PSPL

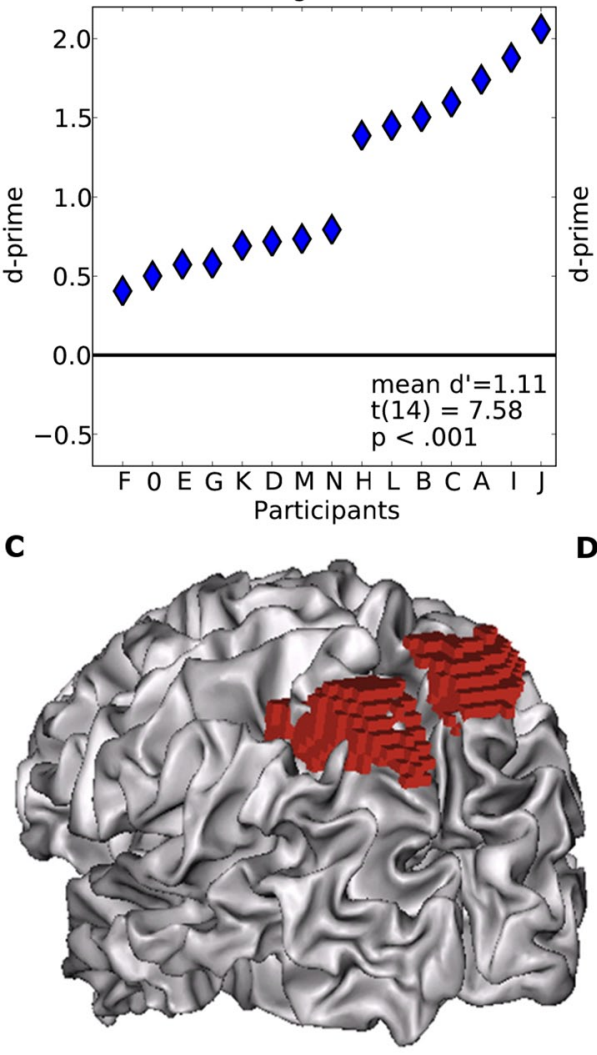

B

Generalization from șaccades to calculation in region PSPL

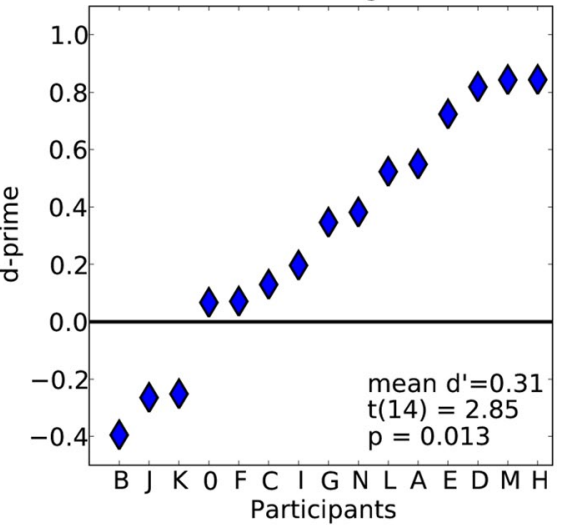

D

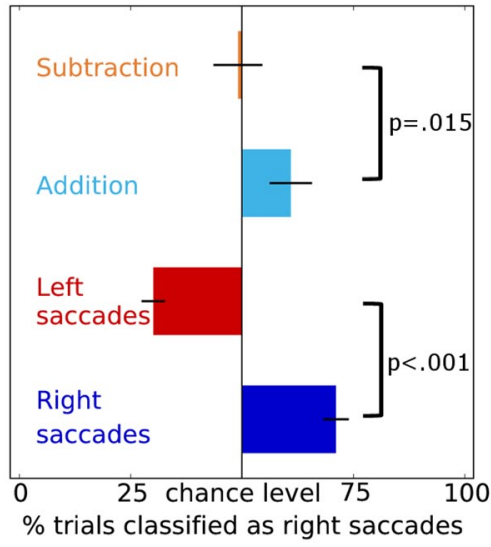

